\title{
THE USE OF MULTIMEDIA IN CHINESE LANGUAGE TEACHING AT BINUS UNIVERSITY
}

\section{印尼建国大学多媒体中文教学}

\author{
Fu Ruomei; Ma Feng \\ Chinese Department, Faculty of Humanities, BINUS University, \\ Jln. Kemanggisan Ilir III No. 45, Kemanggisan - Palmerah, Jakarta Barat 11480, \\ ruomeifu@hotmail.com; mafengde_2009@hotmail.com
}

\begin{abstract}
Article outlines how multimedia is used in the classroom in the Chinese department of Bina Nusantara University. The Binusmaya system standardizes teaching and online communication. Multimedia is an effective supplement to traditional classroom based-teaching of Chinese. Multimedia can increase the learning motivation and improve teaching efficiency. Multimedia also plays a very important role in improving classroom based-teaching of Chinese, teaching standards and enhances a closer relationship between teachers and students.
\end{abstract}

Keywords: Multimedia, Chinese Language Teaching

\section{摘要}

印尼建国大学的多媒体中文教学主要包括多媒体的课堂运用、Binusmaya 网络系统规范教学及 课外网络反馈交流。多媒体的使用对传统教学法是有效的补充, 增强了学习的趣味性, 提高了 教学效率。建国大学中文系的多媒体应用在促进课堂教学、教学规范化及加强师生互动等方面 都发挥了重要作用。

关键词：多媒体，中文教学，建国大学

『1本文被收入第七届中文电话教学研讨会论文集《数字化对外汉语教学实践与反思》, 数据有更新。 


\section{引言}

印尼建国大学是印尼高校中文系中规模最大的一所大学。建国大学中文系成立于 2002 年, 至今已经培养出六届毕业生共 411 人。通过师生的共同努力, 中文系教学规模不断扩大, 学生 中文学习成效显著。在日常教学中, 教师很注重多媒体资源的使用。努力做到依靠科技促进中 文学习, 已取得初步效果。中文系多媒体教学在促进课堂教学、教学规范化及加强师生互动等 方面都发挥了重要作用。

\section{多媒体的课堂运用}

多媒体的使用能有效补充传统教学, 提升教学效率, 提高学生的积极性、主动性与参与精 神。以更贴近学生的方式传授知识, 符合时代的发展方向。李宝贵（2005）认为多媒体有优美 的文字、绚丽的图画、动听的音乐, 它可以尽可能地吸收电影、电视、动画、音响等艺术表现 手法, 把枯燥无味的学习过程转化为赏心悦目的 “娱乐” 活动, 真正做到寓教于乐。

建国大学教学设施先进, 每间教室都配有电脑、投影仪等电教设备。这些设备为实现课堂 多媒体教学提供了技术保障。教师可通过制作幻灯片、播放 DVD 等手段辅助教学。幻灯片的作 用在中国地理课的教学中体现得尤为充分。直观的画面减少了地理术语带来的理解困难, 有利 于激发学生的学习兴趣, 从而有效地提高课堂效率。在其他课程中, 幻灯片的作用也不可小觑。 以教学技巧课程为例, 教师授课时将课程重点制作成幻灯片, 帮助学生理解吸收理论内容, 同 时也有利于知识点的归纳总结。通过播放教学实况影片, 可以加强学生的感性认识, 为学生模 仿教学提供生动的第一手资料。幻灯片的音乐、动画功能在教学中也得到了充分的使用。笔者 在文学课讲解苏轻的《水调歌头》时, 就通过为幻灯片插入三种不同风格的音乐文件, 表现词 的音乐美。一是高中音乐鉴赏曲目《水调歌头》，舒缓有致的典雅美; 二是邓丽君的《但愿人 长久》, 古今交融的甜美感; 三是王菲的《但愿人长久》, 现代磁性的穿透力。美妙的音乐欣 赏中, 感受 “起舞弄清影” 的孤寂, 却又有种翩翩起舞的冲动, 舞蹈的鬼力在音乐旋律中缓缓 流淌。这种形式很受学生的欢迎, 教学效果令人满意。建国大学设有多媒体听力教室, 先进的 设备与形象生动的教学软件相结合, 打破了传统听力教学枯燥单调的教学模式。听力教室可以 让学生有更多机会接触真实语境下的汉语听说内容, 帮助学生提高听力水平, 教师授课也更方 便。特别是在 HSK 考试的模拟练习中, 多媒体听力教室发挥了重要作用。

实践证明多媒体形式教学有利于学生对知识的吸收与运用。作为课堂教学的延伸, 可将所 学知识应用于实践中。王菲的《但愿人长久》, 就在学生自编自演的舞剧《嫦娥奔月的传说》 中得到了充分运用。无论是参加表演的同学, 还是观看的观众, 在乐曲声中重温了课堂学习的 知识。知识在实践中的应用也提高了学生的学习兴趣, 增强了学习的主动性。

\section{Binusmaya 网络教学系统}

作为新时代的综合性大学, 建国大学十分重视电脑网络技术在教学中的作用, 实行网络教 学与传统课堂教学相结合的教学模式。e-Learning 是指通过因特网或其他数字化内容进行学习与 
教学的活动, 它充分利用现代信息技术所提供的、具有全新沟通机制与丰富资源的学习环境, 实现一种全新的学习方式; 这种学习方式将改变传统教学中教师的作用和师生之间的关系, 从 而根本改变教学结构和教育本质 (何克抗. 2001)。建国大学于 2000 年开始使用 IBM 公司开发的 Learning Space 5 (LS5) 网络学习软件系统。中文系于 2003 年开始尝试网络教学时, 使用的 就是 LS5 系统。实践证明, 该系统不能满足学校的教学需求。基于建国大学具有的技术优势, 学校决定自主研发一套符合自身需求的网络教学系统, 即 Binusmaya 网络系统。2004 年底 Binusmaya 系统正式投入使用。经过不断完善, Binusmaya 系统现已相对成熟, 在中文系教学中 的重要性也不断提高。Binusmaya 在教学规范化、加强师生互动等方面发挥了重要作用。 Binusmaya 与授课直接相关的有两大板块：课程资源板块和师生互动论坛。

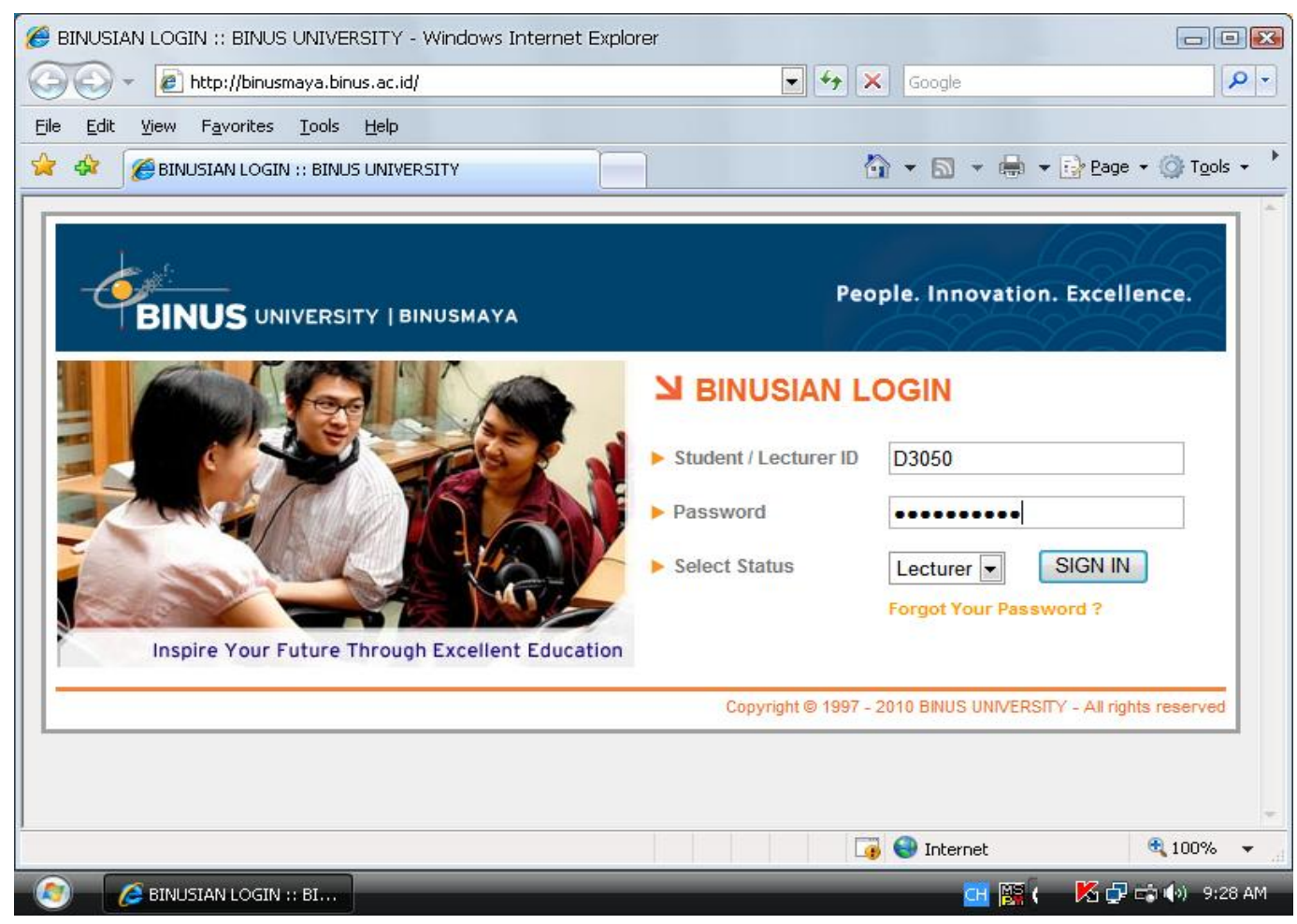

图 1、Binusmaya 登录界面（www. binusmaya. binus. ac. id）

\section{Binusmaya 网络课程资源板块}

Binusmaya 网络课程资源板块记录有所授课程的详细资料。中文系 $80 \%$ 以上的课程为 MCL (Multi Channel Learning) 课程。MCL 要求所有课程内容以 SAP-MP、C0 形式存储 在 Binusmaya 系统中。此模式要求教师在设计课程时, 将每门课教学目标、重点等都录 入专门设计的教学模板中, 上传到 Binusmaya, 同时也为相同课程的任课教师设定了教 学的标准, 做到课程的规范统一。 


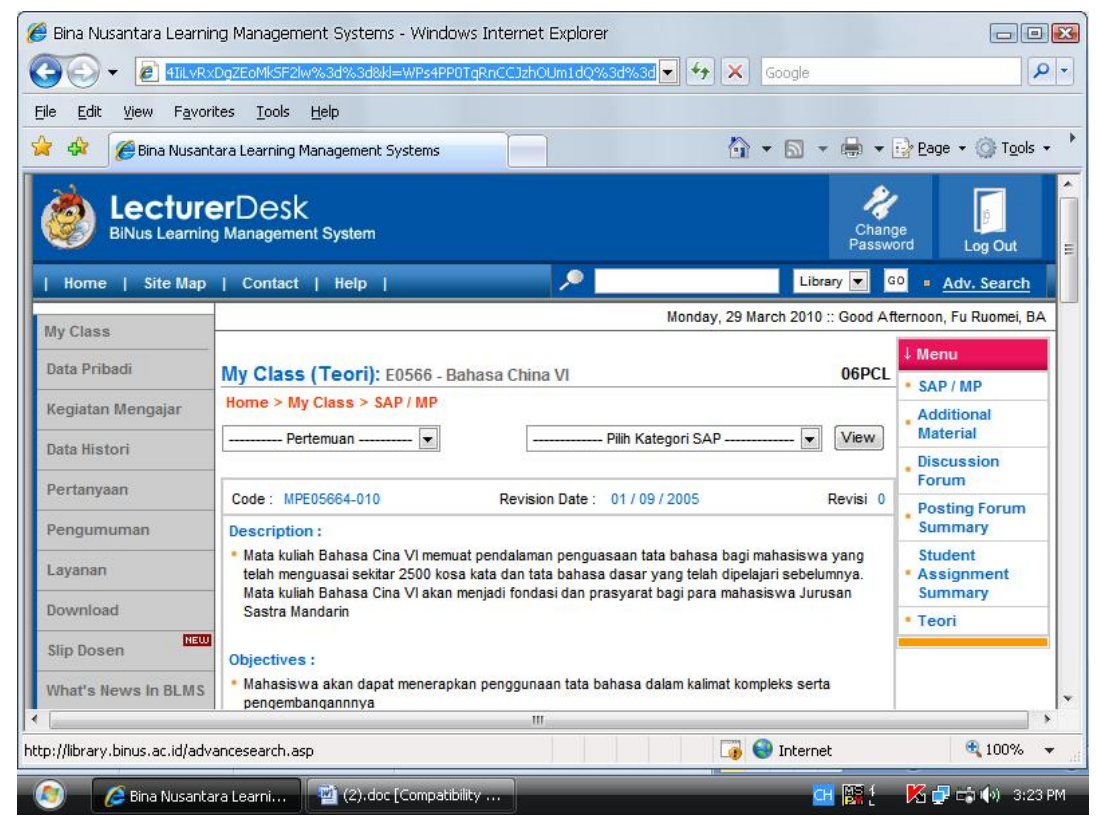

图二、Binusmaya 课程资源界面

SAP（Satuan Acara Perkuliahan）统一授课标准, 规定了每门课的教学资料、考核范围、 考试题型; 期中、期末及平时成绩在总成绩中所占比例等内容, 保证了课程重点的统一。MP (Module Plan) 即课程大纲, 明确了课程的教学目的, 规范了每节课的具体教学目标及授课内 容。CO (Course Outline) 为授课要点, 作为教师授课的参考。每节课都采用幻灯片形式将本 课学习的重点难点内容进行展示, 以便学生的预习与复习。目前中文系课程基本实现了电子化, 课程资料按学期存储在 Binusmaya 系统中, 供师生随时查询。教师上课只需登录 Binusmaya 便 可得到所有授课资料。Binusmaya 的课程内容板块将每节课的内容具体化, 并提供参考资料、 教学建议, 保证了教学内容的一致性和规范化。

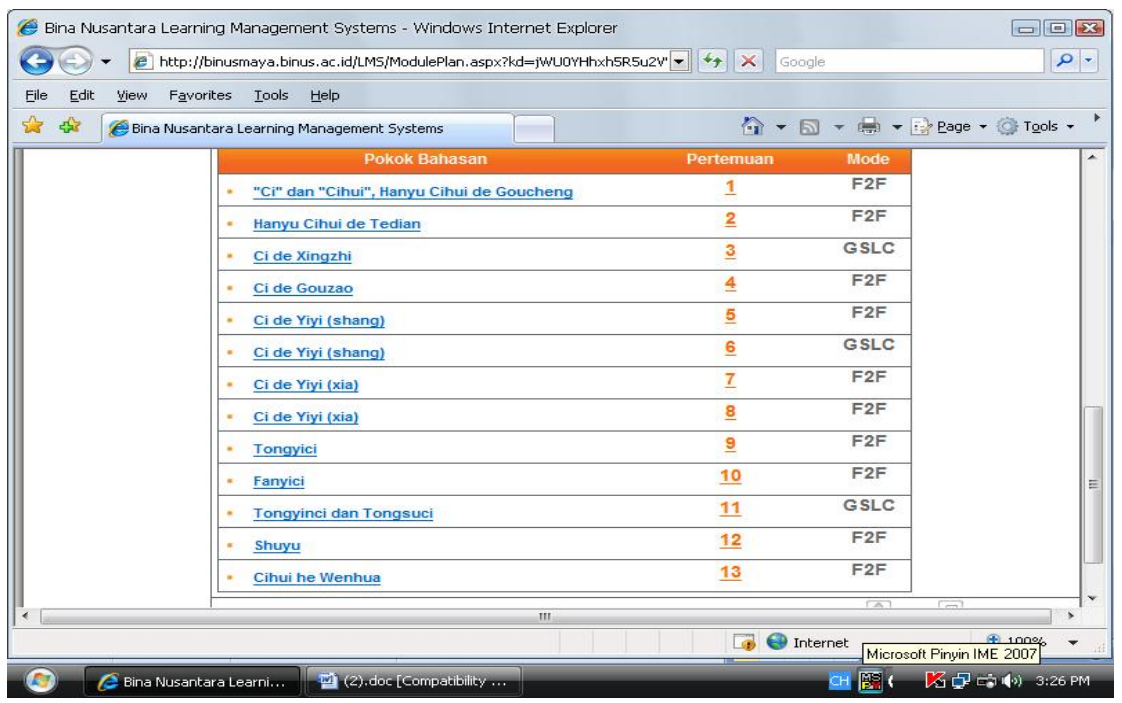

图三、Binusmaya 授课计划界面 


\section{Binusmaya 师生互动论坛}

“启发式指导一一有意义发现” 教学模式中教师的指导行为可分为提问、讲解; 学生的发 现行为可分为自学教材、思考问题、教师的提问、做练习作业、课堂讨论。(陈厚德, 2008) 建国 大学在汉语教学中十分注重启发式指导。MCL 课程都设有 GSLC (Guided Self Learning Class) 自主学习课堂。MCL 课程要求学生每学期至少应有两课时自主学习。教师预先安排好学习内容, 提供相关材料或链接。GSLC 自主学习课堂时, 学生不必到校上课, 而是在 Binusmaya 师生论坛 中就本课内容与教师和同学进行线上沟通。通过阅读资料, 网络查询等手段自主学习, 并完成 教师预留的作业。GSLC 课程的目的在于培养学生的自学能力, 满足学生的不同学习需求。师生 论坛为教师和学生提供了在线交流平台。通过线上互动, 为学生提供平等的学习平台, 使每名 学生都有机会提出自己的观点或疑问。通过论坛, 教师还可以在线批改作业、解答学生疑问、 预告课程安排等。论坛线上沟通方式有利于减少面对面交流时学生的紧张感。学生可以更从容 地表达自己的意见、提出疑问。论坛对整个班级开放, 班级成员都可以看到论坛内容。论坛交 流不但可以加强师生间沟通, 而且也为学生之间交流学习经验、相互借鉴提供便利。

\begin{tabular}{|c|c|c|c|c|c|c|c|c|}
\hline \multicolumn{7}{|c|}{ Bina Nusantara :: Forum - Windows Internet Explorer } & \multicolumn{2}{|c|}{$\square$ 回国 } \\
\hline \multicolumn{4}{|c|}{ 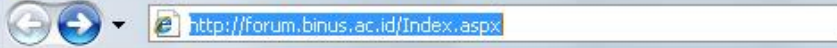 } & $\nabla$ & 4 & Google & \multicolumn{2}{|c|}{ o. } \\
\hline \multicolumn{9}{|c|}{ Eile Eddit Yiew Fagvorites Iools Help } \\
\hline \multicolumn{4}{|c|}{ is the Bina Nusantara Learning Man... } & $\Longrightarrow$ & \multicolumn{4}{|c|}{ 合 - } \\
\hline \multicolumn{6}{|c|}{ (2) LecturerDesk } & $\sum_{\text {Help }}$ & Close & \\
\hline \multicolumn{9}{|c|}{ Forum Diskusi } \\
\hline \multicolumn{9}{|c|}{$\begin{array}{l}\text { E0566 Bahasa China VI } \\
\text { 06PCL }\end{array}$} \\
\hline \multirow{2}{*}{\multicolumn{2}{|c|}{$\begin{array}{l}\text { Jump To } 06 \mathrm{PCL} \text { - E0566 - Bahasa China VI } \\
\begin{array}{|c|c}\text { Topic } \\
\end{array}\end{array}$}} & \multicolumn{2}{|c|}{$\square$ NewTopic } & \multirow{2}{*}{\multicolumn{2}{|c|}{ 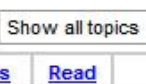 }} & \multicolumn{2}{|r|}{ Page $1-$ of 2} & \\
\hline & & Author & Replies & & & Last Post & & \\
\hline 0 & 第一届青年、学生组散文创作比赛 目 & $\underline{\mathrm{D} 3050}$ & 0 & & 28 & $\begin{array}{l}20 \text { March } 201009: 32 \\
\text { by: } \underline{\text { D3050 }}\end{array}$ & 道渞宙 & \\
\hline$\square$, & Lidya - 第208页的作业。自 & $\underline{1100011314}$ & 1 & & 25 & $\begin{array}{l}20 \text { March } 201009: 28 \\
\text { by: } \underline{03050}\end{array}$ & 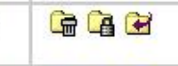 & \\
\hline$\square$ & JUVI/邹露踞 自 & $\underline{1100018075}$ & 1 & & 12 & $\begin{array}{l}20 \text { March } 201009: 24 \\
\text { by: } \underline{03050}\end{array}$ & 道曽 8 & \\
\hline$\square$ & Meriana $06 \mathrm{pcl} 1100003823$ 自 & $\underline{1100003823}$ & 1 & & 10 & $\begin{array}{l}20 \text { March } 201009: 19 \\
\text { by: } 03050\end{array}$ & 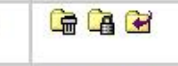 & \\
\hline 0 & Wilsan 谢岸升 110001506006 pcl 第208页的作业 目 & $\underline{1100015060}$ & 1 & & 18 & $\begin{array}{l}20 \text { March } 201009: 12 \\
\text { by: } \underline{03050}\end{array}$ & 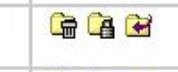 & \\
\hline$\square$ & juliana,06PCL, 1100012903， bab 12 自 & 1100012903 & 1 & & 9 & $\begin{array}{c}18 \text { March } 201016: 10 \\
\text { by: } \underline{3050}\end{array}$ & 渞曾 & \\
\hline \multicolumn{9}{|c|}{ |" III } \\
\hline & & & & & & (2) Internet & $\oplus_{100 \%}$ & $\checkmark$ \\
\hline
\end{tabular}

图四、Binusmaya 师生论坛界面 


\section{网络考勤监督教学进程}

自 2009 年 9 月起, 中文系实行网上考勤。建国大学的每间教室都设有电子打卡感应器, 教 师和学生提前 5 分钟进入教室, 通过电子卡考勤, 师生信息及打卡时间会在 Binusmaya 考勤系 统中显现。授课结束后, 教师登录 Binusmaya 考勤页面, 师生对当天出勤名单进行核对, 同时 选择一名学生监督。考歏面与预先设定的教学计划页面已经建立了链接, 本课时授课要点会 在考勤页面出现。教师应在考歏面教学内容一栏填写本课时实际授课内容, 学生可以判断出 教师是否按照预定内容授课。教师输入本节课授课内容, 师生确认无误后, 由学生代表输入密 码确认, 完成网络考勤。为保证考勤的有效性, 考勤页面只能在教室登录进入。出席学生名单 及本课时授课内容必须由教师和学生共同确认完成, 保证了考勤的公正性。网上考勤不仅考查 教师学生出席情况, 而且通过内部链接, 将考勤与授课 SAP-MP 教学内容联系在一起, 考勤的 同时也是对本节课所学内容的回顾总结, 并对教师授课起到监督作用。

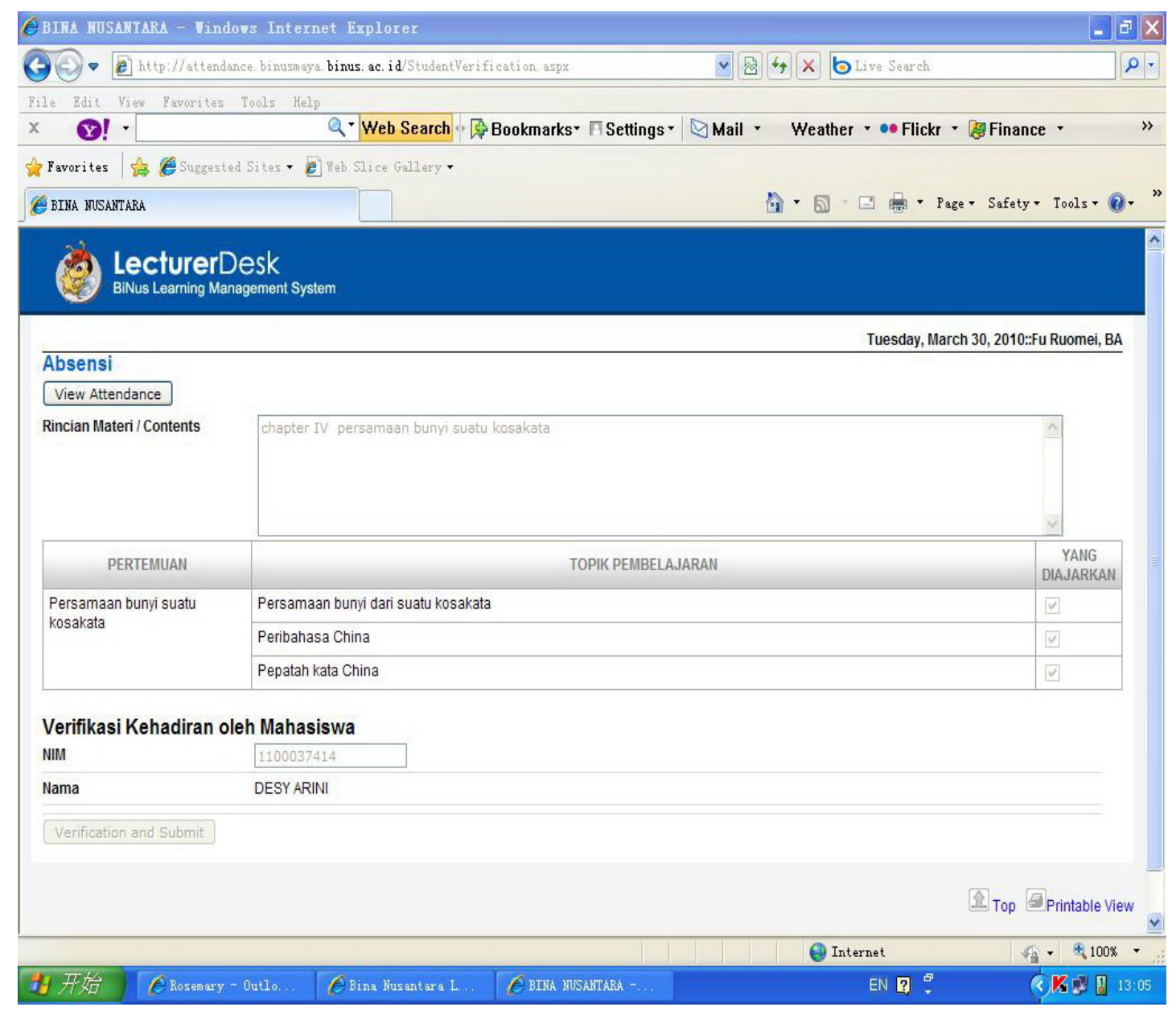

图五、Binusmaya 网络考勤界面 


\section{课外网络反馈交流}

随着时代的发展, 网络应用越来越广泛, “网络博客” 慢慢成为一种抒写情怀的空间。通 过 Facebook、MSN 等网络空间, 可以和学生实时交流。多种渠道反馈, 让课外交流成为实践教 学的第二课堂与有益补充。近年来网络交友系统 Facebook 在学生中十分流行。建国大学鼓励教 师拥有自己的 Facebook, 作为与学生建立良好师生关系、加强交流的渠道, 同时也是课堂教学 的延伸。实践证明, 在 Facebook 中与学生沟通更容易, 效果更明显。Binusmaya 论坛的师生互 动还是紧紧围绕课程进行的。Facebook 则突破了课程的局限。在这一空间中没有师生之别, 大 家是以朋友的身份相互沟通。在本文的书写过程中, 有关电脑截图部分的操作, 笔者就曾通过 Facebook 寻求学生的帮助。

通过 Facebook 教师能了解到学生的真实想法, 可以与学生更深入的交流。教师多关注学生 的动态, 对学生遇到的问题表示关切, 在学生有需求时予以指导和帮助。双向互动不但拉近了 师生距离, 而且作为课堂教学的延伸, 可以在潜移默化中对学生施以影响。以写作课课外延伸 教学为例。曾经有学生在 Facebook 博客以《幸福在哪里? 》为题表达心声: “我累了, 怎么努 力也无法得到幸福……幸福是什么呢? 在生活中, 遇到各种各样的困难是一定的事情。怎么能 面对这种困难呢? 我总是想, 在生活中的事情是没完没了的。我有这个力量来面对它吗? 什么 时候才能愉快地过日子呢? 怎么才能得到幸福呢? 为什么这么难? 我想, 全世界的人最需要 的, 那就是幸福。幸福, 看起来是一个很简单的事情, 但是实际上, 它是一种最难追到的事情。 困难总是一直困扰着我们。我们怎么解脱它，它也不要离开。我必须放弃了这个小小的希望吗？”

这段真情实感的流露, 充满了迷茫与无助。笔者感慨之余回复: “幸福在哪里? 幸福就在 我们身边! 世界不是缺少幸福, 而是缺少发现幸福的目光! 青草的幸福是雨露的滋养, 绿树的 幸福是阳光的爱抚, 碧海的幸福是游鱼的缤纷, 蓝天的幸福是白云的可爱。你的幸福是什么呢? 幸福不代表拥有: 追求梦想的艰辛, 亲朋好友的愉悦, 青春美好的时光……这一切都充满了幸 福！拥有一颗美丽、善良、充满爱的心，你就拥有了整个世界。……用心，享受生活的点滴！ 用爱，精心构筑理想的宏伟蓝图！用美，追寻彼此的目光与绚烂多姿的世界！”

教学反馈需要师生间的交流, 如果教师在写作教学中真正同学生互动起来, 其效果是显著 而有效的。写作不能只是学生的独角戏, 也需要教师发挥创作才思。教师的以身作则, 写作示 范有助于拉近与学生的距离。充分利用每一个机会, 激发学生的写作热情。虽然例文只是学生 的牢骚之语, 但却展现了学生的写作才华。教师的及时反馈, 不仅是对学生自觉写作行为的鼓 励, 也有助于学生感悟能力的提高。其他学生可以在博客里看到教师的回复, 相互的探讨评论 将会营造出一种轻松愉快的氛围，让写作不再枯燥无味，师生间的交流更自然有效。 


\section{结语}

多媒体中文教学尚在起步阶段, 建国大学中文系在教学中对多媒体应用进行多种形式的探 索。通过教学实践, 不断改革教学方法, 力求通过有效运用多媒体促进学生的中文学习。教师 以学生的兴趣为切入点, 将先进的网络技术引入传统教学中, 运用多渠道电化教学, 积极寻求 有效的教学模式。师生课内外的网络互动, 是课堂教学的有效补充, 有助于所学知识的㺬固、 师生感情的融洽。

\section{参考文献}

陈厚德．有效教学．北京：教育科学出版社，2008 (8)：134

何克抗. E-Learning 与高校教学的深化改革. 2002 年 1 月 11 日 http://www. chinaet. org/tg/1lelearning/2244. htm

李宝贵. 对外汉语教学及汉语本体研究. 北京：北京大学出版社, 2005 (5)：153

马峰. 幸福在哪里? 雅加达: 印尼国际日报, 2009 年 7 月 26 日

马峰. 快乐写作初探. 第一届 “华文作为第二语言之教与学” 国际研讨会光盘. 新加坡华文教 研中心，2009（11）

许丽妮、马峰. 对外汉语教学中的 Power Point 应用. 多维视野下的对外汉语教学研究. 桂林: 广西师范大学出版社, 2009（7)：352 